\title{
Synthesis of substituted Coumarin derivatives and their spectral Characterization
}

\author{
Neha Mishra, Vrushali Patil, Ashish Asrondkar, A.S Bobade, \\ Abhay Chowdhary \\ ${ }^{I}$ Department of Chemotherapy, Haffkine Institute for Training, Research and Testing, India
}

\begin{abstract}
A series of some coumarin derivative have been synthesized by the interaction of 4-hydroxy coumarin with substituted benzaldehyde. The identities of these synthesized compounds have been established on the basis of chemical transformation and IR, ${ }^{1} H$ NMR and Mass spectral studies. In the present study synthesized compounds were studied for their in-vitro anti inflammatory activity by using HRBC membrane stabilization method, all compounds studied shows satisfactory results
\end{abstract}

Keywords: Coumarin, Anti-inflammatory, etc

\section{Introduction}

Inflammatory diseases are becoming common in aging society throughout the world. Recent studies indicate that the mediators and cellular effectors of inflammation are important constituents of the local environment of tumors ${ }^{1}$. Inflammation in the body's response to noxious or injurious stimuli, characterized by warmth, redness of the skin, pain, swelling and loss of function. It is a part of host defense mechanism. There are several tissue factors that are known to be involved in the inflammatory reactions such as release of histamines, bradykinin and prostaglandins ${ }^{2 .}$ Coumarins (2H-1-benzopyran-2-ones) are important oxygen containing fused heterocycles used in drugs and dyes ${ }^{3}$. Coumarins be bound their class name to 'coumarou' the vernacular name of the Tonka bean (Dipteryx odorata willd, Fabaceae), from which coumarin itself was isolated in $1820^{4}$. They are the family of lactones containing benzopyrone skeletal framework that have enjoyed isolation from plant as well as total synthesis in the laboratory ${ }^{5}$. The incorporation group as a fused component into parent coumarin alters the property of parent coumarin and converts it into a more useful product ${ }^{6}$. Coumarin is plant flavonoids widely distributed in nature. Natural coumarins are known to have antidiabetic activity ${ }^{7}$, anabolic antioxidant and hepato protective activities ${ }^{8}$. Substituted coumarins derivatives have been reported to have variety of biological activities. The potent antibiotics like Novobiocin, Coumaromycin and Chartesium are coumarin derivatives. Recently, the interest on these compounds has been revived owing to their use as fluorescent markers in the biochemical determination of enzymes. Introduction of fluoro and sulfonamide moieties into coumarin side chain hoping for an improvement of biological activity because incorporation of fluorine to various heterocycles is known to influence the biological activity ${ }^{9}$ and the sulfonamide moiety itself possesses important antibacterial ${ }^{10}$ and antitumor activity ${ }^{11}$. Specifically 1, 5 substituted benzothiazepine ${ }^{12}$ are well known compounds for diverse therapeutically properties ike antimicrobial ${ }^{13}$, antihypertensive ${ }^{14}$, calcium channel blocker ${ }^{15}$, blood platelet aggregation inhibitory ${ }^{16}$ and coronary vasodilatory effects ${ }^{17}$. In the present study, we have evaluated the anti-inflammatory and antibacterial activity of some newly synthesized coumarin derivatives.

\section{Materials and methods}

All raw materials used in the synthesis have been obtained from M/S Fluka AG (Bachs, Switzerland) and M/S Sigma-Aldrich chemicals and Co. Inc. (Milwoukee, WI,USA). Melting points were recorded on a Thermonik Melting point Apparatus (Campbell Electronics, Mumbai, India) and are uncorrected. IR spectra were recorded on a IR-Affinity, Shimadzu using DRS system. ${ }^{1} \mathrm{H}-\mathrm{NMR}$ spectra have been recorded on a JEOL AL-300 FT-NMR spectrometer (300 MHz, JEOL Ltd., Tokyo, Japan), using TMS as internal standard in solvent DMSO. Masss datahave been recorded on Agilent GC-MS Elemental analysis has been carried out on a C, H, and N Elemental Analyzer (Thermo-Finnigan Flash EA 1112, Italy).

\subsection{Experimental}

1.1.1 Preparation of ethyl [(2-0xo-2H-chromen-4-yl)oxy] acetate (Compound 1)

4-Hydroxy Coumarin $(2.0 \mathrm{~mol})$ in dry acetone was dissolved and Potassium carbonate $(1.0 \mathrm{~mol})$ was added, the reaction mixture was refluxed for 6 hours and then Ethychlorolacetate was added and the reaction mixture was refluxed for another 6 hours the reaction mass then was neutralized by using glacial acetic acid and then extraction was given by using diethylether. The completion of the reaction was mentioned by TLC. Yield 


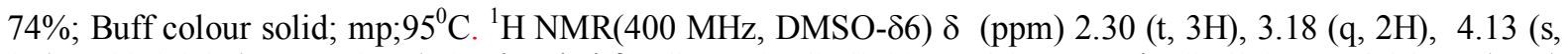
$2 \mathrm{H})$, 7.18-8.26 (m, 5H, Ar-H) Anal. calcd for $\mathrm{C}_{13} \mathrm{H}_{12} \mathrm{O}_{5}: \mathrm{C}, 62.90 ; \mathrm{H}, 4.87$; Found: C, 62.37; H, 4.16. IR (KBr) $\mathrm{cm}^{-1}: \quad 2943\left(-\mathrm{CH}_{3}\right), 1719(\mathrm{C}=\mathrm{O}), 1214(\mathrm{C}-\mathrm{O}) . \mathrm{MS}(\mathrm{m} / \mathrm{z}): 248\left[\mathrm{M}^{+}\right]\left(\mathrm{C}_{13} \mathrm{H}_{12} \mathrm{O}_{5}^{+}\right), 203\left(\mathrm{C}_{11} \mathrm{H}_{7} \mathrm{O}_{4}\right), 175\left(\mathrm{C}_{10} \mathrm{H}_{7} \mathrm{O}_{3}\right)$, $145\left(\mathrm{C}_{9} \mathrm{H}_{5} \mathrm{O}_{2}\right)$.

\subsubsection{PREPARATION OF 2-[(2-OXO-2H-ChrOMEN-4-YL)OXY]ACETOHYDRAZIDE(COMPOUND 2)}

Compound 1(1 mol) dissolve in ethanol treated with mixture of hydrazine hydrate hydrochloride solution ( $1 \mathrm{~mol}$ ) was refluxed for $6 \mathrm{hrs}$. The reaction was cooled, poured into ice cold water. Solid product was filtered, dried and Recrystallized from ethanol. Yield $72 \%$; brown colour solid; mp; $168^{0} \mathrm{C} ;{ }^{1} \mathrm{H}$ NMR $(400 \mathrm{MHz}$, DMSO- $\delta 6) \delta(\mathrm{ppm})$ 6.98-7.65 (m, 5H Ar-H), 10.35 (s, 1H), 5.89 (s, 2H), 4.12 (s, 2H) Anal. calcd for $\mathrm{C}_{11} \mathrm{H}_{10} \mathrm{~N}_{2} \mathrm{O}_{4}: \mathrm{C}, 56.41 ; \mathrm{H}, 4.30 ; \mathrm{N}, 11.96$ Found: C, 56.37; H, 4.16; N, 11.40. IR (KBr) cm ${ }^{-1}$ : $1645(\mathrm{C}-\mathrm{O}-\mathrm{C})$, 2953(-CH3), 1720(C=O), $3342(\mathrm{~N}-\mathrm{H}), \mathrm{MS}(\mathrm{m} / \mathrm{z}): 234\left[\mathrm{M}^{+}\right]\left(\mathrm{C}_{11} \mathrm{H}_{10} \mathrm{~N}_{2} \mathrm{O}_{4}^{+}\right), 218\left(\mathrm{C}_{11} \mathrm{H}_{8} \mathrm{NO}_{4}\right), 203\left(\mathrm{C}_{11} \mathrm{H}_{7} \mathrm{O}_{4}\right)$, $175\left(\mathrm{C}_{10} \mathrm{H}_{7} \mathrm{O}_{3}\right), 145\left(\mathrm{C}_{9} \mathrm{H}_{5} \mathrm{O}_{2}\right)$

\subsubsection{PREPARATION OF 4-[(5-METHOXY-1,3-BENZOTHIAZOL-2-YL)SULFANYL]-2H-CHROMEN-2-ONE (COMPOUND 3A) \\ Compound 2(1 mol) dissolve in ethanol to this substituted Benzaldehyde $(1 \mathrm{~mol})$ few drops of glacial} acetic acid was added and refluxed for $2 \mathrm{hrs}$. The reaction was cooled, poured into ice cold water. Solid product was filtered, dried and Recrystallized from ethanol.; ${ }^{1} \mathrm{H}$ NMR(400 MHz, DMSO- $\delta 6$ ) $\delta$ (ppm) 7.12-8.16 (m, $10 \mathrm{H} \mathrm{Ar}-\mathrm{H}), 3.18(\mathrm{~s}, 2 \mathrm{H}), 5.34(\mathrm{~s}, 1 \mathrm{H}), 2.84(\mathrm{~s}, 1 \mathrm{H})$ Anal. calcd for $\mathrm{C}_{18} \mathrm{H}_{14} \mathrm{O}_{4} \mathrm{~N}_{2}: \mathrm{C}, 67.07 ; \mathrm{H}, 4.38 ; \mathrm{N}, 8.69$. Found: C, 67.37; H, 4.16; N,8.60IR (KBr) cm ${ }^{-1}: 1615(\mathrm{C}-\mathrm{O}-\mathrm{C}), 2943(-\mathrm{CH}), 1745(\mathrm{C}=\mathrm{O}), 3344(\mathrm{~N}-\mathrm{H}) . \mathrm{MS}(\mathrm{m} / \mathrm{z}): 322$ $\left[\mathrm{M}^{+}\right]\left(\mathrm{C}_{18} \mathrm{H}_{14} \mathrm{O}_{4} \mathrm{~N}_{2}^{+}\right), 245\left(\mathrm{C}_{12} \mathrm{H}_{9} \mathrm{~N}_{2} \mathrm{O}_{4}\right), 218\left(\mathrm{C}_{11} \mathrm{H}_{8} \mathrm{NO}_{4}\right), 203\left(\mathrm{C}_{11} \mathrm{H}_{7} \mathrm{O}_{4}\right), 175\left(\mathrm{C}_{10} \mathrm{H}_{7} \mathrm{O}_{3}\right), 145\left(\mathrm{C}_{10} \mathrm{H}_{5} \mathrm{O}_{2}\right)$

2.1.4 Preparation of $\mathrm{N}^{\prime}$-[(E)-(4-chlorophenyl)methylidene]-2-[(2-ox0-2H-chromen-4yl)oxy]acetohydrazide (Compound 3b) ${ }^{1} \mathrm{H}$ NMR(400 MHz, DMSO-d6) d (ppm) 7.22-8.36 (m, 19H Ar-H), 3.56 (s, 2H), $5.84(\mathrm{~s}, 1 \mathrm{H})$, $2.94(\mathrm{~s}, 1 \mathrm{H})$ Anal. calcd for $\mathrm{C}_{18} \mathrm{H}_{14} \mathrm{~N}_{2} \mathrm{O}_{3} \mathrm{~S}: \mathrm{C}, 63.89 ; \mathrm{H}, 4.17, \mathrm{~N}, 8.28$; Found: C, 63.81; H, 4.16. N, 8.82 IR $(\mathrm{KBr}) \mathrm{cm}^{-1}$ 2943(-CH3), $1745(\mathrm{C}=\mathrm{O}), 1214(\mathrm{C}-\mathrm{O})$

MS (m/z): $356\left[\mathrm{M}^{+}\right]\left(\mathrm{C}_{18} \mathrm{H}_{13} \mathrm{~N}_{2} \mathrm{O}_{4} \mathrm{Cl}^{+}\right), 321\left(\mathrm{C}_{18} \mathrm{H}_{13} \mathrm{~N}_{2} \mathrm{O}_{4}\right), 245\left(\mathrm{C}_{12} \mathrm{H}_{9} \mathrm{~N}_{2} \mathrm{O}_{4}\right), 218\left(\mathrm{C}_{11} \mathrm{H}_{8} \mathrm{NO}_{4}\right), 203\left(\mathrm{C}_{11} \mathrm{H}_{7} \mathrm{O}_{4}\right)$, $175\left(\mathrm{C}_{10} \mathrm{H}_{7} \mathrm{O}_{3}\right), 145\left(\mathrm{C}_{10} \mathrm{H}_{5} \mathrm{O}_{2}\right)$

\subsubsection{Preparation of 4N'-[(E)-(4-fluorophenyl)methylidene]-2-[(2-oxo-2H-chromen-4-} yl)oxy] acetohydrazide (Compound 3c)

${ }^{1} \mathrm{H}$ NMR(400 MHz, DMSO- $\delta 6$ ) $\delta(\mathrm{ppm}) 7.36-8.26$ (m, 9H Ar-H), 3.10 (s, 2H), 5.44 (s, 1H), $2.01(\mathrm{~s}, 1 \mathrm{H})$ Anal. calcd for $\mathrm{C}_{18} \mathrm{H}_{13} \mathrm{~N}_{2} \mathrm{O}_{4} \mathrm{~F}: \mathrm{C}, 63.53 ; \mathrm{H}, 3.85 ; \mathrm{N}, 8.23$; Found: $\mathrm{C}, 63.72 ; \mathrm{H}, 3.16, \mathrm{~N}, 8.79$

IR $(\mathrm{KBr}) \mathrm{cm}^{-1}: 2943(-\mathrm{CH} 3), 1785(\mathrm{C}=\mathrm{O}), 1294(\mathrm{C}-\mathrm{O})$

MS (m/z): ): $340\left[\mathrm{M}^{+}\right]\left(\mathrm{C}_{18} \mathrm{H}_{13} \mathrm{~N}_{2} \mathrm{O}_{4} \mathrm{~F}^{+}\right), 321\left(\mathrm{C}_{18} \mathrm{H}_{13} \mathrm{~N}_{2} \mathrm{O}_{4}\right), 245\left(\mathrm{C}_{12} \mathrm{H}_{9} \mathrm{~N}_{2} \mathrm{O}_{4}\right), 218\left(\mathrm{C}_{11} \mathrm{H}_{8} \mathrm{NO}_{4}\right), 203\left(\mathrm{C}_{11} \mathrm{H}_{7} \mathrm{O}_{4}\right)$, $175\left(\mathrm{C}_{10} \mathrm{H}_{7} \mathrm{O}_{3}\right), 145\left(\mathrm{C}_{10} \mathrm{H}_{5} \mathrm{O}_{2}\right)$

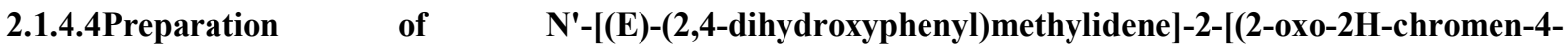 yl)oxy] acetohydrazide (Compound 3d) \\ ${ }^{1} \mathrm{H}$ NMR(400 MHz, DMSO- $\delta$ 6) $\delta(\mathrm{ppm}) 7.02-8.60(\mathrm{~m}, 8 \mathrm{H} \mathrm{Ar}-\mathrm{H}), 3.28(\mathrm{~s}, 2 \mathrm{H}), 5.41(\mathrm{~s}, 1 \mathrm{H}), 2.94(\mathrm{~s}, \quad 1 \mathrm{H})$, $6.34(\mathrm{~s}, 1 \mathrm{H}), 6.74(\mathrm{~s}, 1 \mathrm{H})$ Anal. calcd for $\mathrm{C}_{18} \mathrm{H}_{14} \mathrm{~N}_{2} \mathrm{O}_{6}: \mathrm{C}, 61.02 ; \mathrm{H}, 3.98, \mathrm{~N}, 7.91$; Found: $\quad \mathrm{C}, 61.17$; H, 3.46. N, 7.32 IR $(\mathrm{KBr}) \mathrm{cm}^{-1}: 3235(-\mathrm{NH}), 1725(\mathrm{C}=\mathrm{O}), 1394(\mathrm{C}-\mathrm{O})$ \\ MS (m/z): ): $354\left[\mathrm{M}^{+}\right]\left(\mathrm{C}_{18} \mathrm{H}_{14} \mathrm{~N}_{2} \mathrm{O}_{6}{ }^{+}\right), 337\left(\mathrm{C}_{18} \mathrm{H}_{13} \mathrm{~N}_{2} \mathrm{O}_{5)} 321\left(\mathrm{C}_{18} \mathrm{H}_{13} \mathrm{~N}_{2} \mathrm{O}_{4}\right), 245\left(\mathrm{C}_{12} \mathrm{H}_{9} \mathrm{~N}_{2} \mathrm{O}_{4}\right), 218\left(\mathrm{C}_{11} \mathrm{H}_{8} \mathrm{NO}_{4}\right)\right.$, $203 \quad\left(\mathrm{C}_{11} \mathrm{H}_{7} \mathrm{O}_{4}\right), 175\left(\mathrm{C}_{10} \mathrm{H}_{7} \mathrm{O}_{3}\right), 145\left(\mathrm{C}_{10} \mathrm{H}_{5} \mathrm{O}_{2}\right)$}

\subsubsection{Preparation of $\mathrm{N}^{\prime}-[(\mathrm{E})$-(4-nitrophenyl)methylidene]-2-[(2-oxo-2H-chromen-4-}

${ }^{1} \mathrm{H}$ NMR(400 MHz, DMSO- $\delta$ 6) $\delta(\mathrm{ppm}) 7.32-8.56(\mathrm{~m}, 9 \mathrm{H} \mathrm{Ar}-\mathrm{H}), 3.01(\mathrm{~s}, 2 \mathrm{H}), 5.04(\mathrm{~s}, 1 \mathrm{H}), 2.04(\mathrm{~s}, \quad 1 \mathrm{H})$ Anal. calcd for $\mathrm{C}_{18} \mathrm{H}_{13} \mathrm{~N}_{3} \mathrm{O}_{6}: \mathrm{C}, 58.86 ; \mathrm{H}, 3.57, \mathrm{~N}, 11.44$; Found: $\mathrm{C}, 58.17 ; \mathrm{H}, 3.46 . \mathrm{N}, \quad 11.32$. IR $(\mathrm{KBr}) \mathrm{cm}^{-1}$ : $3235(-\mathrm{NH}), 1725(\mathrm{C}=\mathrm{O}), 1394(\mathrm{C}-\mathrm{O}) \mathrm{MS}(\mathrm{m} / \mathrm{z}):): 367\left[\mathrm{M}^{+}\right]\left(\mathrm{C}_{18} \mathrm{H}_{13} \mathrm{~N}_{3} \mathrm{O}_{6}{ }^{+}\right), 321\left(\mathrm{C}_{18} \mathrm{H}_{13} \mathrm{~N}_{2} \mathrm{O}_{4} \quad\right), \quad 245$ $\left(\mathrm{C}_{12} \mathrm{H}_{9} \mathrm{~N}_{2} \mathrm{O}_{4}\right), 218\left(\mathrm{C}_{11} \mathrm{H}_{8} \mathrm{NO}_{4}\right), 203\left(\mathrm{C}_{11} \mathrm{H}_{7} \mathrm{O}_{4}\right), 175\left(\mathrm{C}_{10} \mathrm{H}_{7} \mathrm{O}_{3}\right), 145 \quad\left(\mathrm{C}_{10} \mathrm{H}_{5} \mathrm{O}_{2}\right)$ 
Table 1: Schematic Representation of Titled Compounds<smiles>CCOC(=O)CCl</smiles>

4-hydroxy-2H-chromen-2

ethyl

-one

chloroacetate

DryAcetone

$\mathrm{K}_{2} \mathrm{CO}_{3}$<smiles>CCOC(=O)COc1cc(=O)oc2ccccc12</smiles>

ethyl

[(2-oxo-2H-chromen-4-yl)oxy]acetat<smiles>CCCCN</smiles>

2-[(2-oxo-2H-chromen-4-yl)oxy]acetohy drazide<smiles>[R]c1cccc(/C=N/NC(=O)COc2cc(=O)oc3ccccc23)c1</smiles>

2-[(2-oxo-2H-chromen-4-yl)oxy]- $N$ '-[(Z)-phenylmethylidene]acetohydrazide

Table 2: Substitution table

\begin{tabular}{|l|c|c|c|c|c|}
\hline Compounds & Substitution & Yield & M.P/B.P & Nature & Colour \\
\hline $3 \mathrm{a}$ & $\mathrm{H}$ & 60 & 116 & Crystalline & White \\
\hline $3 \mathrm{~b}$ & $4-\mathrm{Cl}$ & 72 & 176 & Amorphorous & buff \\
\hline $3 \mathrm{c}$ & $4-\mathrm{F}$ & 77 & 182 & Amorphorous & white \\
\hline $3 \mathrm{~d}$ & $2,4-\mathrm{Di} \mathrm{OH}$ & 56 & 119 & Amorphorius & Pale yellow \\
\hline $3 \mathrm{e}$ & $4-\mathrm{NO}_{2}$ & 64 & 189 & liquid & Reddis brown \\
\hline
\end{tabular}


Table 3: Substitution table Anti- inflammatory results \%inhibition

\begin{tabular}{|c|c|c|c|c|c|c|c|c|c|c|}
\hline Concentration & 3a & \%inh & 3b & \%inh & 3c & \%inh & 3d & \%inh & 3e & \%inh \\
\hline 6.25 & 0.370 & 68.80 & 0.415 & 76.61 & 0.400 & 77.46 & 0.288 & 75.71 & 0.242 & 79.59 \\
\hline 12.5 & 0.357 & 69.89 & 0.305 & 82.81 & 0.352 & 80.16 & 0.263 & 77.82 & 0.200 & 83.13 \\
\hline 25 & 0.314 & 73.52 & 0.301 & 83.04 & 0.349 & 80.33 & 0.256 & 78.41 & 0.196 & 83.47 \\
\hline 50 & 0.272 & 77.06 & 0.294 & 83.44 & 0.342 & 80.73 & 0.227 & 80.86 & 0.184 & 84.48 \\
\hline 100 & 0.255 & 78.49 & 0.288 & 83.77 & 0.338 & 80.95 & 0.214 & 81.95 & 0.162 & 86.34 \\
\hline Positive control & 1.186 & & 1.775 & & 1.775 & & 1.186 & & 1.186 & \\
\hline
\end{tabular}

\section{Results}

The activity of coumarin derivatives was studied for in vitro anti-inflammatory Activity by HRBC membrane stabilization method. Among all the concentrations $100 \mu \mathrm{g} / \mathrm{ml}$ showed significant anti-inflammatory activity and $86.34 \%$ protection of HRBC in hypotonic solution. Results were compared with standard Diclofenac which showed $90 \%$ protection. The Coumarin derivative exhibited membrane stabilization effect by inhibiting hypotonicity induced lyses of RBC membrane. The RBC membrane is analogous to the lysosomal membrane and its stabilization implies that the extract may as well stabilize lysosomal membranes. Stabilization of lysosomal membrane is play an important role in limiting the inflammatory response by preventing the release of lysosomal constituents of activated neutrophile such as bactericidal enzymes and proteases, which cause further tissue inflammation and damage upon extra cellular release

\section{Conclusion}

In vitro anti-inflammatory study reveals that the activity of the drugs under test was satisfactory as compared to that of standard DFS i.e. the coumarin derivatives were found out to be potent enough to suppress haemolysis. However, the \% inhibition of haemolysis in case of DFS treated RBC's was slightly high as compared to that of the $\%$ inhibition provided by the test drugs.

Also within the series of test drugs, (i.e. various coumarin derivatives) those which were substituted with electron donating groups showed an enhancement of anti-inflammatory activity while those derivatives which were formed by substituting an electron withdrawing moiety were found to be showing a drastic reduction of anti-inflammatory activity.

\section{Acknowledgements}

The authors are thankful to SAIF, IIT, Powai, Mumbai for carrying out the elemental analysis (CHN) and also thankful to SAIF, Patiala University, Punjab for recording the NMR spectra.

\section{References}

[1]. A. Mantovani, Allavena P, Sica A, Nature, 2008, 454: 436-444

[2]. R. S. Chavan, H. N. More, A. V. Bhosale, Int J Pharm Biomed Res, 2010, 1(4):135-143.

[3]. S. Rajasekaran, G. K. Rao, S. P. N. Pai, A. Ranjan, International journal of chem tech research, 2011, 3(2): 555-559.

[4]. S. D. Nachiket, R. P. Shashikant, S. S. Dengale, D. S. Musmade, M. Shelar, V. Tambe, M. Hole, Der Pharma Chemica, 2010, 2(2): 65-71.

[5]. A. O. Olayinka, N. C. Obinna, Journal of Heterocyclic Chemistry, 2010, 47:179-187.

[6]. D. I. Brahnbhatt, J. M. Gajera, V. P. Pandya, M. A. Patel, Ind. J. chem. 2007, 46(B):869-871.

[7]. R. Sharma, V. Arya, J Chem. Pharm. Res. 2011, 3(2):204-212.

[8]. R. D. H. Murrey, D. Medez, S. A. Brown, The natural coumarins occurrences, chemistry and biochemistry, John Wiley Interscience, Newyork 1982 F. Zoni, P.Vicini, Arch. Pharm. 1998, 331:219.

[9]. H. Yoshino, N. Ueda, J. Niijima, H. Sugumi, Y. Kotake, N. Koyanagi, K. Yoshimatsu, M. Asada, T. Watanabe,

[10]. T. Nagasu, K. Tsutahara, A. Lijima, K. Kitoh, J. Med. Chem. 1992, 35:2496.

[11]. R. P. Jignesh, D. T. Jignasu, D. K. Chintan, D. R. Kishor, ARKIVOC 2008, 12:233-244.

[12]. B. K. Karal, V. P. Chavan, A. S. Mame, R. V. Hangarage, Korean J. Med. Chem. 2000, $10,84$.

[13]. K. Kinoshita, A. Mitani, J. D. Hearse, V. M. Braimbridge, S. H. Manning, J. Surg.Res. 1989, $97: 166$.

[14]. J. Belusa, V. Hruskova, Z. Haas, Z. Kaminska, F. Picha, J. Dusek, M. Trefulka, V. Kysilka, V. Wojnar, Chem.

[15]. Abstr. 1992, 118: 2459706.

[16]. M. Padwad, V. N. Ingle, J Indian chem. Soc. 1999, 76:161.

[17]. A. G. Nikalje, R. D. Ingle, V. E. Bhingolikar, K. A. Mane, Indian J. Heterocycl. Chem. 2003, 13: 33.

[18]. Z. Hua, J. Geo, L. Zhu-bo, M. Bu-Hyun, S. D. Soo, G. Manjunath, ARKIVOC 2008, 233-44. 